\title{
A NEWLY DEVELOPED FIB SYSTEM FOR TEM SPECIMEN PREPARATION
}

\author{
T.Kamino*, T.Yaguchi*, Y.Kuroda *, T.Hashimoto **, T.Ohnishi**, T.Ishitani**, \\ K.Umemura***, K.Asayama**** \\ *Hitachi Science Systems, Ltd., 882 Ichige, Hitachinaka, Ibaraki, 312-8504 Japan \\ ** Hitachi High-Technologies Corp., 882 Ichige, Hitachinaka, Ibaraki, 312-8504 Japan \\ *** Central Research Laboratory, Hitachi, Ltd., Kokubunji, Tokyo 185-8601 Japan \\ ****Semiconductor and Integrated Circuits Division, Hitachi, Ltd., Oume, Tokyo, 198-8512 Japan
}

In the materials characterization using transmission electron microscope(TEM), FIB technique is demanding more and more as the method to prepare electron transparent specimen ${ }^{1)}$. We have developed a dedicated FIB system FB-2000A employing FIB-TEM(STEM) compatible specimen stage $^{2)}$ and an FIB micro-sampling tehnique ${ }^{3-5)}$. The FIB-TEM(STEM) compatible specimen stage allowed site specific TEM specimen preparation with a positional accuracy of $0.1 \mu \mathrm{m}$ or better ${ }^{2}$. The FIB micro-sampling allowed extraction of TEM specimen directly from bulk sample without any pre-FIB preparation.

Recently, an FIB system FB-2100 with a newly designed 40kV ion optics has been developed to perform high-speed TEM specimen preparation. Accelerating voltage is variable from $10 \mathrm{kV}$ to $40 \mathrm{kV}$ at a minimum step of $5 \mathrm{kV}$. The maximum ion beam current and maximum ion beam current density of the new ion optics are $30 \mathrm{nA}$ and $25 \mathrm{~A} / \mathrm{cm}^{2}$, respectively. The ion milling speed in TEM specimen preparation is approximately twice as high as that of previous model(FB-2000A). No noticeable increase of beam damage for the TEM specimen preparation at $40 \mathrm{kV}$ has been confirmed. Figure 1 shows a high resolution TEM image of Si single crystal specimen prepared at $40 \mathrm{kV}$. Crystal lattice and the dumb-bell structure with the spacing of $0.136 \mathrm{~nm}$ are clearly observed. The requirements for a high-speed TEM specimen preparation are rapidly increasing. One of solutions is employment of automatic fabrication for rough milling of a TEM specimen. We have developed a new automatic fabrication system employing a new marker detection unit based on a phase only correlation(POC) method. The marker detection unit worked for various brightness and sharpness of the images. Figure 2 shows a series of test marker images observed at various images brightness. Images 2-8 were allowable in the marker detection. Figure 3 shows a series of test images observed at various Z-position. All markers in these images were identified as the memorized marker. The newly designed ion optics allowed reduction of ion beam diameter together with increase of ion beam current. Minimum probe size and the probe current for the milling are $12 \mathrm{~nm}$ and $0.02 \mathrm{nA}$, respectively. Additionally, some optimization have been made in settings of voltages, lens for various sizes and accelerating voltages. Those improvements were effectively worked out to improve scanning ion microscopy(SIM) image quality. The SIM image resolution is $6 \mathrm{~nm}$ or better at $40 \mathrm{kV}$.

\section{References}

1. T.Ishitani, et al., J. Electron Microsc.43, 5 (1994)322-326

2. T.Kamino, et al., Proc. Microsc. and Microanal. 6, Supp.12 (2000) 510-511

3. K.Umemura, et al., Electron Microscopy, 34, Supp. 1 (1999) 510-511

4. T.Ohnishi,et al., Proc. $25^{\text {th }}$ Int.Symp. for Testing and Failure Analysis (1999) 449-503 
5. T.Yaguchi, et al., Proc. Microsc. and Microanal.7, Supp. 2 (2001) 938-939

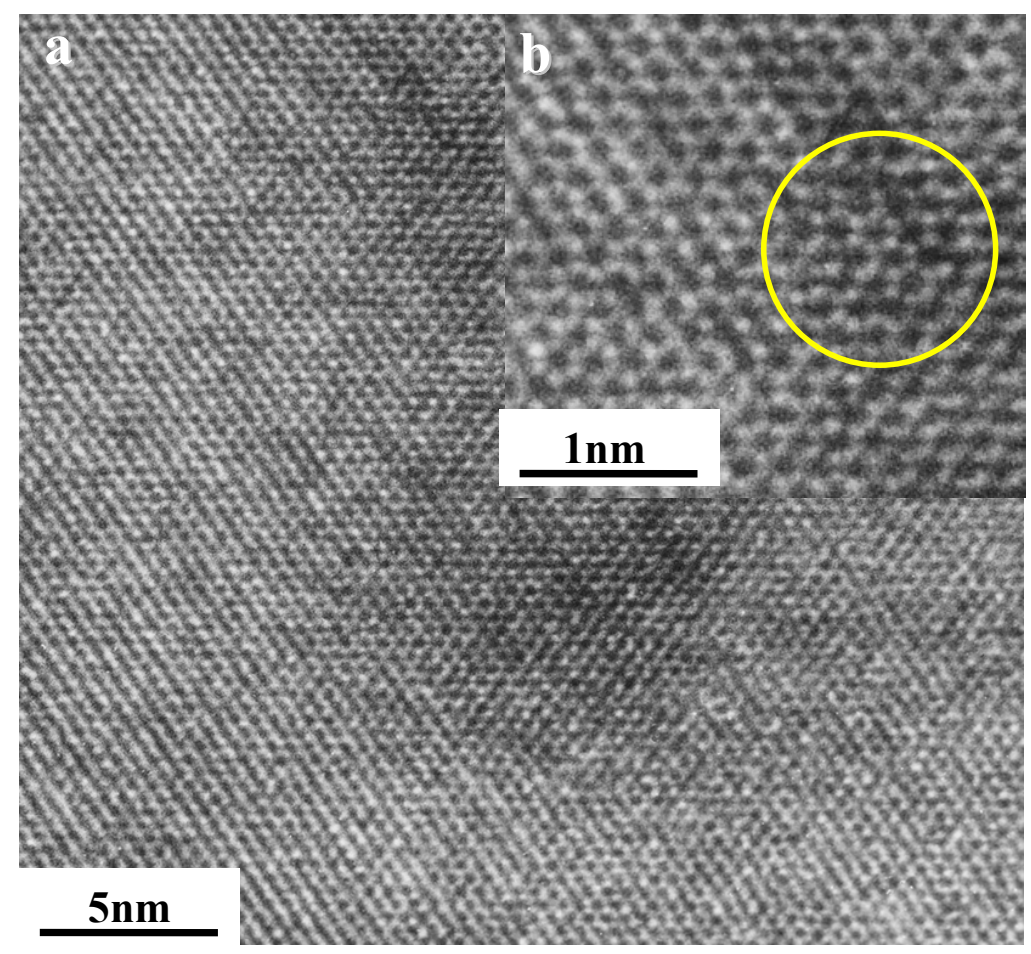

Figure 1. Crystal lattices image (a) and the dumb-bell structure(b) observed on a $\mathrm{Si}$ single crystal specimen prepared by FIB milling at $40 \mathrm{kV}$.

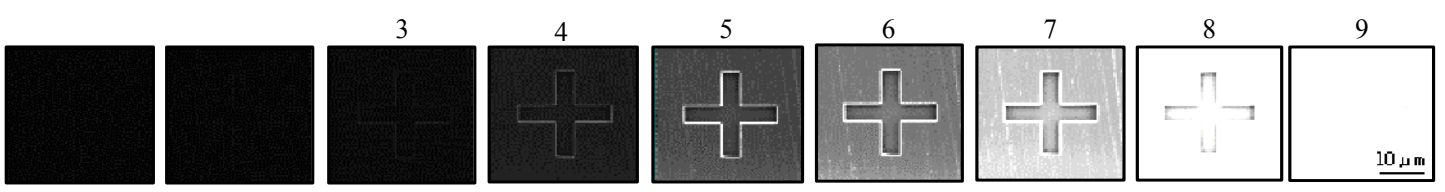

Figure 2. A series of SIM images of a cross marker observed at various brightness.

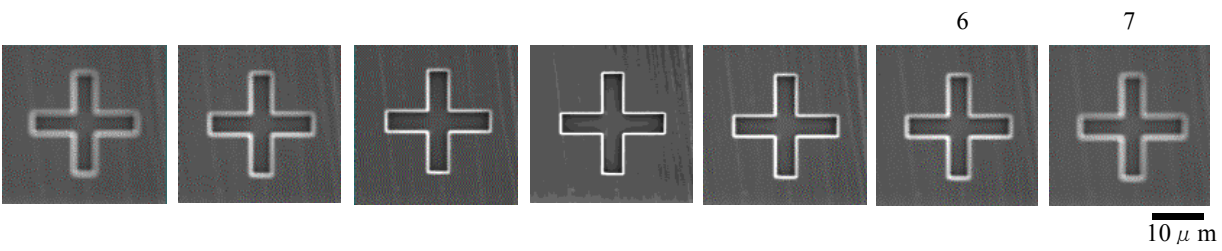

Figure 3. A series of SIM images of a marker at various Z-position. All markers in these images were identified as the memorized marker. 